\title{
Autonomic Nervous System Derangement as a Predictor of Cardiovascular Disease in Obese Postmenopausal Women
}

\author{
Madhuri Taranikanti ${ }^{1} \quad$ Akhila Dronamraju ${ }^{1} \quad$ Sudha Bala ${ }^{2}$ Rohith Kumar Guntuka ${ }^{1}$ \\ Aswin Kumar Mudunuru ${ }^{3}$ \\ ${ }^{1}$ Department of Physiology, ESIC Medical College, Sanathnagar, \\ Hyderabad, India \\ 2Department of Community Medicine, ESIC Medical College, \\ Sanathnagar, Hyderabad, India \\ ${ }^{3}$ Psychophysiology Lab, Asha Hospital, Hyderabad, India

\begin{abstract}
Address for correspondence Madhuri Taranikanti, MD, Department of Physiology, ESIC Medical College, Sanathnagar, Hyderabad-38, Telangana, India (e-mail: madhuri.tarani@gmail.com; madhuri.taranikanti@gmail.com).
\end{abstract}

Ind J Car Dis Wom 2019;4:8-14

\begin{abstract}
Keywords

- cardiovascular disease

- autonomic functions

- postmenopausal women

- estrogen
\end{abstract}

Background and Aim Cardiovascular disease is more prevalent in women after menopause and particularly in those aged $>60$ years. Obesity during menopause has become a growing cause of concern as the source of estrogen during this period is extragonadal. Autonomic function tests serve as effective markers of autonomic activity. Any derangement of autonomic functions in obese postmenopausal women would serve as an early marker for the detection of cardiovascular disease. The aims and objectives of this study were to measure autonomic functions tests in obese postmenopausal women and to correlate the autonomic activity with anthropometric parameters and serum estrogen levels.

Material and Methods A cross-sectional study of 101 postmenopausal women aged $>45$ years was undertaken. Women with heart disease, diabetes mellitus, hypertension, and respiratory disorders were excluded from this study. A digital data acquisition system, Lab Chart Pro 8.0 was used to perform the autonomic function tests.

Results Parasympathetic activity decreased significantly with higher body mass index (BMI). Sympathetic activity that includes diastolic blood pressure and blood pressure measured after isometric handgrip test significantly increased with BMI. A strong negative correlation existed between BMI and parasympathetic high-frequency (HF) domain index. There was a strong positive correlation between BMI and serum estradiol levels.

Conclusion Measuring the autonomic activity in obese postmenopausal women along with anthropometric indices and estrogen levels would provide strong evidence of a potential target for existing as well as future heart disease. Any autonomic dysfunction, if detected early, would enable early interventions to prevent heart disease.

\section{Introduction}

Cardiovascular diseases (CVDs) are one of the leading causes of death in women across the globe. ${ }^{1}$ The Registrar General of India projected CVD as the most important cause of death in women. ${ }^{2}$ A large proportion of the elderly population in whom the percentage of heart disease is high is constituted by women due to their longer life expectancy. ${ }^{3}$ CVD is more prevalent in women after menopause and particularly in those aged $>60$ years with more deaths occurring annually due to CVD, a fact that remains unrecognized. ${ }^{4}$ Obesity is one of the recognized risk factors for heart disease, and its prevalence 
Autonomic Nervous System Derangementin in Postmenopausal Women Taranikanti et al. 9

is continuing to increase globally. ${ }^{5}$ Menopause has become a growing cause of concern as obesity, and metabolic syndromes are common during this period in women. ${ }^{6}$ Genetic, hormonal, and exogenous factors are responsible for changes in body composition during menopause. A decline in the levels of estrogens during menopause is responsible for these changes. Women in the postmenopausal period have a higher risk of developing obesity that in turn helps conversion of peripheral androgens to estrogens including estradiol (E2). ${ }^{7}$ Unfortunately, these estrogens do not provide cardiovascular protection. Autonomic functions affecting the heart are disturbed in the postmenopausal period and obesity. Measurement of cardiac autonomic functions may prove to be a useful tool for diagnosing heart diseases in obese postmenopausal women. High body fat percentage is associated with autonomic dysregulation, with marked sympathetic overactivity and inhibition of parasympathetic activity. Any autonomic dysfunction, if detected early, would enable early interventions to prevent heart diseases. Hence, measuring the autonomic activity in postmenopausal women along with anthropometric indices and estrogen levels would provide strong evidence of a potential target for existing as well as future heart disease in these women. Very few studies in this field are available from the Indian subcontinent, particularly, South India, and hence, this study was taken up with the following objectives:

- To stratify women in the postmenopausal period into three categories of nonobese, overweight, and obese, based on the anthropometric parameters

- To measure the body mass index (BMI), total body fat percentage, and waist-to-hip ratio (WHR) in postmenopausal women, and correlate with the autonomic function tests

- To correlate serum estrogen levels with autonomic functions

\section{Material and Methods}

- Study design: Cross-sectional study.

- Study area: Cardiovascular physiology.

- Study period: May to July 2018.

- Study participants: 101 postmenopausal women aged > 45 years.

- Exclusion criteria: Women with evidence of CVDs, diabetes mellitus, hypertension, and respiratory diseases.

- Procedure: A cross-sectional study of 101 postmenopausal women was conducted who did not show any gross systemic disease. After measuring their height, weight, and BMI, the women were divided into three groups (group I nonobese, group II overweight, and group III obese). Height was measured using a stadiometer that was rounded off to the nearest centimeter. A digital body fat analyzer (AVON Corp.) was used that measured weight and body fat percentage using bioelectric impedance technique. All women with $\mathrm{BMI}<25$ were included in group I and labeled as nonobese who served as controls, those with BMI between 25 and 30 were included in group II and labeled as overweight, and those with BMI > 30 were included in group III and labeled as obese. Both groups were matched for age and menopausal duration. Waist measurement was taken at the level of iliac crest in centimeters. Hip circumference was taken at the fullest point around buttocks. WHR of this study participants was determined. WHR $>0.9$ was considered to be obese. Basal blood pressure (BP) measurement and autonomic function tests (AFTs) were performed using a data acquisition system, PowerLab, Lab Chart Pro 8/35 (ADInstruments Pty Limited) applying the standard protocols as per the manufacturer's guidelines. The system includes transducers to record various parameters. Pressure transducer that is used to record BP also records pressure during Valsalva's maneuver. Electrocardiographic (ECG) leads were connected in lead II through the biopotential transducer. Force transducer was used to record the isometric handgrip performance. These transducers are used to test cardiac sympathetic and parasympathetic functions. By use of chemiluminescence method to correlate with the autonomic functions, $2 \mathrm{~mL}$ of venous blood was collected to measure the serum estrogen levels. Written informed consent was taken from all the participants of this study, and the institute's ethics committee permission was obtained.

\section{Autonomic Function Tests}

- Tests to assess parasympathetic activity include

- Heart rate variability (HRV) to postural change

- HRV to deep breathing

- HRV to Valsalva's maneuver

- Tests to evaluate sympathetic activity include

- BP response to postural change

- BP response to isometric handgrip test

All the participants were made to rest for 15 minutes in a comfortable supine position before starting the AFTs. Between any two tests, a resting time of about 5 minutes was given.

\section{Heart Rate Response to Postural Change (30:15)}

A baseline ECG was recorded in lead II for at least 2 minutes in all the women, and then the patients were instructed to stand erect, when told, as quickly as possible while ECG recording was continuing. The longest $\mathrm{R}-\mathrm{R}$ interval at 30th beat and the shortest R-R interval at 15 th beat were noted, and 30:15 ratio was determined. The ratio was considered normal if it was $\geq 1.04$, and any value $\leq 1.00$ was considered abnormal.

\section{Heart Rate Response to Deep Breathing}

All the patients were instructed to take deep inspiration followed by expiration for six times over a period of 1 minute making the duration of each inspiration and each expiration 5 seconds completing six respiratory cycles in 1 minute. At least three to five practice sessions were given to the patients before the actual recording was done to make them familiar and comfortable with the procedure so as to perform the test with maximum accuracy possible to maintain the timing of each cycle. The mean of maximum heart rate during inspiration and minimum heart rate during expiration was measured, and the difference was calculated. The normal values should be $>10$ to 15 beats/min. ${ }^{8}$ Expiratory-inspiratory (E:I) 
ratio is one of the indicators and is the ratio of the longest $R-R$ interval during expiration to the shortest R-R interval during inspiration and should be $>1.2 .^{9}$

\section{Heart Rate Variation to Valsalva's Maneuver}

Baroreceptor function is evaluated by Valsalva's maneuver. Patients were instructed to exhale forcefully through a 5 - or $10-\mathrm{mL}$ disposable syringe used as a mouthpiece that was connected to the pressure transducer to reflect the pressure rise during forceful expiration. The participants were asked to maintain the pressure at $40 \mathrm{~mm} \mathrm{Hg}$ for 15 seconds. During the procedure, ECG recording was done and was continued for at least 30 seconds after the procedure. ${ }^{10}$ The ratio of the longest $\mathrm{R}$ - $\mathrm{R}$ after expiration to the shortest R-R during forceful expiration was calculated. Normal values should be $\geq 1.21$, abnormal $\leq 1.1{ }^{11}$

\section{Blood Pressure Response to Change in Posture}

Blood pressure was recorded after resting for at least 5 minutes in supine position. Then, the patient was asked to stand up erect and the BP was recorded immediately. ${ }^{11}$ The difference between systolic blood pressures (SBP) recorded during supine and standing postures was calculated. A fall in SBP by $>20 \mathrm{~mm} \mathrm{Hg}$ and a fall in diastolic blood pressure (DBP) by $>10 \mathrm{~mm} \mathrm{Hg}$ were considered abnormal. ${ }^{8}$

\section{Blood Pressure Response to Sustained Isometric Handgrip}

Patients were asked to maintain the force at one-third of maximum force determined during calibration for 5 minutes. The maximum force for each patient differed. The dominant hand was used for this purpose. Immediately after releasing the pressure on the transducer at the end of 5 minutes, BP measurements were taken in the other arm. The highest DBP immediately after the procedure was noted, and the average diastolic pressure at rest was calculated. The difference between the two should normally be $>15 \mathrm{~mm} \mathrm{Hg}{ }^{8}$

\section{Heart Rate Variability Domains}

The two main approaches to analyze HRV are the timedomain and frequency domain in which only normal beats are included and abnormal beats are excluded. The square root of variance between normal to normal beats (SDNN or SDRR) is widely used as an index of the time domain. ${ }^{12}$ To identify specific components of variability, it was split into various frequency components forming the frequency domain that uses the power spectral density analysis. ${ }^{13-15}$ Parasympathetic activation is faster and short lasting due to exhaustion of neurotransmitter acetylcholine whereas sympathetic activity lasts little longer. Time-domain analysis using standard deviation of NN (SDNN or SDRR) (normal-to-normal intervals) describes the overall HRV as it assesses both long- and short-term variabilities. On the other hand, root mean square of successive differences (rMSSD) describes only short-term variation reflecting parasympathetic activity. The proportion of differences in consecutive NN intervals $>50$ milliseconds is given by pNN 50\%, and it also signifies short-term variability similar to rMSSD. Frequency-domain indices recorded over short periods include the low-frequency (LF) component (LF 0.04-0.15 $\mathrm{Hz}$ ) that is modulated by sympathetic and parasympathetic and baroreceptor activity. High-frequency (HF) range component, HF between 0.15 and $0.4 \mathrm{~Hz}$ is modulated by the parasympathetic nervous system and is associated with respiration and $\mathrm{BP}$ changes. The ratio of $\mathrm{LF} / \mathrm{HF}$ reflects the presence of interaction between both types of autonomic modulation. The raw signals that were recorded were carefully processed for any artifacts and ectopic beats were duly removed. The Lab Chart computer algorithm can be programmed to automatically exclude such undesirable signals.

\section{Statistical Analysis}

The mean values of all the parameters taken from both the groups were tabulated, and statistical analysis was performed using analysis of variance (ANOVA) to see the variation between the three groups. Student's $t$-test was used to calculate the $p$ value for significance, and correlation between parameters was established. $p<0.05$ was considered significant, and $p<0.01$ was considered highly significant.

\section{Results}

The baseline anthropometric data of all postmenopausal women participating in this study were calculated. The mean age of the women was $53.43 \pm 6.73$ years. The average duration of menopause was $8 \pm 6.55$ years, the mean BMI was $25.73 \pm$ 4.43, the mean WHR was $0.93 \pm 0.13$, and the mean body fat percentage was $32.24 \pm 8.13$. When the women were stratified as per the BMI, it was observed that the duration of menopause was high in overweight and obese women. Though the WHR was $>0.9$ in all the three groups, there was no statistical difference between the three groups. The body fat percentage progressively increased with increasing BMI (-Tables 1,2 ). The AFTs were divided into those that assessed parasympathetic functions and those that assessed sympathetic functions. HRV with respect to posture, deep breathing, and Valsalva's maneuvers was part of the parasympathetic tests. HRV was found to decrease with increasing BMI, with posture, deep breathing, and Valsalva's maneuvers. The least variability was found in obese postmenopausal women. $p$ Value was found to be significant between the three groups for all the parasympathetic tests performed ( - Table 3 ). The parasympathetic tests measured the BP variation with posture and performance of isometric handgrip exercise. The decline in DBP was found to be more significant than that in SBP. There was a significant difference in the DBP after the performance of the isometric handgrip exercise in all the three groups ( - Table 4 ). Our study shows that the time-domain variables are less in the obese group than nonobese postmenopausal women. However, there is no statistical significance between time-domain variables between the two groups (-Table 5). A statistically significant 
Table 1 Baseline anthropometric data of all postmenopausal women participating in the study

\begin{tabular}{|l|l|l|l|}
\hline Parameters & Nonobese $($ mean \pm SD) & Overweight mean (mean \pm SD) & Obese mean (mean \pm SD) \\
\hline Age & $54.02 \pm 6.89$ & $52.94 \pm 6.44$ & $52.9 \pm 7.06$ \\
\hline Menopause duration & $6.93 \pm 5.44$ & $7.66 \pm 6.46$ & $8.29 \pm 6.80$ \\
\hline Height & $1.54 \pm 0.08$ & $1.53 \pm 0.06$ & $1.53 \pm 0.04$ \\
\hline Weight & $51.57 \pm 7.18$ & $63.85 \pm 6.22$ & $75.36 \pm 7.23$ \\
\hline BMI & $21.79 \pm 2.03$ & $27.16 \pm 1.27$ & $32.29 \pm 2.06$ \\
\hline Waist & $84.46 \pm 8.03$ & $96.63 \pm 7.97$ & $103.65 \pm 7.15$ \\
\hline Hip & $91.61 \pm 9.71$ & $103.73 \pm 6.16$ & $114.75 \pm 6.59$ \\
\hline WHR & $0.93 \pm 0.18$ & $0.93 \pm 0.05$ & $0.9 \pm 0.06$ \\
\hline Body fat percentage & $25.96 \pm 5.09$ & $33.89 \pm 4.12$ & $43.79 \pm 3.86$ \\
\hline TBW & $59.75 \pm 3.28$ & $52.9 \pm 2.98$ & $45.40 \pm 4.27$ \\
\hline
\end{tabular}

Abbreviations: BMI, body mass index; SD, standard deviation; TBW, total body water; WHR, waist-to-hip ratio.

Table 2 Anthropometric data of study participants after grouping based on BMI

\begin{tabular}{|l|l|l|l|l|l|}
\hline Parameters & N (Total = 101) & Age $(\mathbf{y})$ & BMI & WHR & Body fat percentage \\
\hline Nonobese & 46 & $54.02 \pm 6.89$ & $21.79 \pm 2.03$ & $0.93 \pm 0.18$ & $25.96 \pm 5.09$ \\
\hline Overweight & 35 & $52.94 \pm 6.44$ & $27.16 \pm 1.27$ & $0.93 \pm 0.05$ & $33.89 \pm 4.12$ \\
\hline Obese & 20 & $52.9 \pm 7.06$ & $32.29 \pm 2.06$ & $0.90 \pm 0.06$ & $43.79 \pm 3.86$ \\
\hline
\end{tabular}

Abbreviations: BMI, body mass index; WHR, waist-to-hip ratio.

Table 3 Heart rate variability (HRV) with posture, deep breathing, and Valsalva's maneuver

\begin{tabular}{|l|l|l|l|l|}
\hline Parasympathetic tests & Normal & Overweight & Obese & $p$ Value \\
\hline $\begin{array}{l}\text { HRV with posture } \\
\text { Supine to standing } \\
30: 15\end{array}$ & $1.1 \pm 0.23$ & $1.02 \pm 0.18$ & $0.95 \pm 0.15$ & 0.028 \\
\hline HRV with deep breathing & $15.23 \pm 7.45$ & $11.09 \pm 7.35$ & $9.06 \pm 5.52$ & 0.003 \\
\hline HRV with Valsalva's maneuver & $1.74 \pm 1.59$ & $1.23 \pm 0.64$ & $1.03 \pm 0.27$ & 0.037 \\
\hline
\end{tabular}

Table 4 BP variations with posture and isometric hand grip

\begin{tabular}{|c|c|c|c|c|}
\hline Sympathetic tests & Normal $(\mathrm{mm} \mathrm{Hg})$ & Overweight (mm Hg) & Obese $(\mathrm{mm} \mathrm{Hg})$ & $p$ Value \\
\hline $\begin{array}{l}\text { BP with posture } \\
\text { (Supine to standing) } \\
\text { Decline in SBP } \\
\text { Decline in DBP }\end{array}$ & $\begin{array}{l}13.16 \pm 12.84 \\
4.35 \pm 13.31\end{array}$ & $\begin{array}{l}16.96 \pm 14.92 \\
11.46 \pm 12.63\end{array}$ & $\begin{array}{l}18.53 \pm 11.17 \\
11.69 \pm 5.13\end{array}$ & $\begin{array}{l}0.269 \\
0.016\end{array}$ \\
\hline $\begin{array}{l}\text { BP with isometric handgrip } \\
\text { (Difference in DBP from rest to post grip) }\end{array}$ & $15.4 \pm 10.29$ & $12.46 \pm 12.66$ & $7.54 \pm 8.21$ & 0.035 \\
\hline
\end{tabular}

Abbreviations: BP, blood pressure; DBP, diastolic blood pressure; SBP, systolic blood pressure.

Table 5 Comparison of time-domain parameters between obese and nonobese groups

\begin{tabular}{|l|l|l|l|l|}
\hline \multicolumn{2}{|l|}{ Time-domain variables } & Nonobese & Obese & $p$ Value \\
\hline 1 & Average HR BPM & $71.75 \pm 6.75$ & $86.26 \pm 2.86$ & 0.049 \\
\hline 2 & SDRR & $48.81 \pm 13.77$ & $31.08 \pm 15.12$ & 0.21 \\
\hline 3 & rMSSD & $35.14 \pm 13.82$ & $29.3 \pm 13.39$ & 0.63 \\
\hline 4 & NN 50\% & $7.86 \pm 11.06$ & $7.84 \pm 6.87$ & 0.99 \\
\hline
\end{tabular}

Abbreviations: HR BPM, heart rate, beats per minute; rMSSD, root mean square of successive differences; SDRR, standard deviation of R-R.

difference was observed between the frequency-domain variables of obese and nonobese postmenopausal women (-Table 6). High HF values indicate dominant parasympathetic activity in nonobese compared with obese women, and high LF values in obese indicate a predominance of sympathetic activity. When the serum E2 was correlated with BMI, a strong 
12 Autonomic Nervous System Derangementin in Postmenopausal Women Taranikanti et al.

positive correlation between the two parameters was found (-Table 7). A strong negative correlation also existed between BMI and parasympathetic HF-domain index (-Table 8). Further, a strong negative correlation was observed between estrogen and HF variable of AFT ( - Table 9; cFig. 1).

\section{Discussion}

Cardiovascular diseases are common with aging and menopausal in women. Indian women have a higher body fat percentage for a given BMI when compared with their western counterparts. Studies have shown that obesity causes accelerated CVD after menopause. ${ }^{16}$ In this study, an increase in anthropometric parameters such as body fat percentage, waist and hip circumferences, and WHR were observed with increasing BMI in obese patients, which is consistent with previous studies. ${ }^{16-20}$ In our study, we also observed that the SBP and DBP were higher in obese women than in the nonobese, which is consistent with other studies done previously. ${ }^{21,22}$

Heart rate variability is less in obese individuals as obesity is associated with marked sympathetic activation and

Table 6 Comparison of frequency-domain parameters between obese and nonobese groups

\begin{tabular}{|l|l|l|l|l|}
\hline S. No & Frequency-domain variables & Nonobese & Obese & $p$ Value \\
\hline 1 & LF & $63.76 \pm 10.6$ & $85.47 \pm 5.89$ & 0.05 \\
\hline 2 & HF & $31.02 \pm 7.29$ & $16.23 \pm 2.95$ & 0.05 \\
\hline 3 & LF/HF & $2.16 \pm 0.84$ & $5.27 \pm 1.5$ & 0.04 \\
\hline
\end{tabular}

Abbreviations: HF, high frequency; LF, low frequency.

Table 7 Correlation between serum estradiol levels and BMI

\begin{tabular}{|l|l|l|l|l|}
\hline Parameters & Nonobese & Overweight & Obese & $r$ Value \\
\hline Serum E2 level (pg/dL) & $5.17 \pm 0.745$ & $11.13 \pm 20.41$ & $39.36 \pm 117.05$ & 0.994 \\
\hline BMI & $21.97 \pm 1.80$ & $26.79 \pm 2.16$ & $32.29 \pm 2.06$ \\
\hline
\end{tabular}

Abbreviations: BMI, body mass index; E2, estradiol.

Table 8 Correlation between BMI and frequency-domain variable of AFT

\begin{tabular}{|l|l|l|l|}
\hline Parameters & Nonobese & Obese & $r$ Value \\
\hline BMI & $21.97 \pm 1.80$ & $32.29 \pm 2.06$ & -1 \\
\hline $\begin{array}{l}\text { HF } \\
\text { Frequency-domain variable }\end{array}$ & $31.02 \pm 7.29$ & $16.23 \pm 2.95$ \\
\hline
\end{tabular}

Abbreviations: AFT, autonomic function test; BMI, body mass index; HF, high frequency.

Table 9 Correlation between E2 and HF

\begin{tabular}{|l|l|l|l|}
\hline Parameters & Nonobese & Obese & $r$ Value \\
\hline E2 levels $(\mathrm{pg} / \mathrm{dl})$ & $5.17 \pm 0.745$ & $39.36 \pm 117.05$ & -1 \\
\hline $\begin{array}{l}\text { HF } \\
\text { Frequency-domain variable }\end{array}$ & $31.02 \pm 7.29$ & $16.23 \pm 2.95$ \\
\hline
\end{tabular}

Abbreviations: E2, estradiol; HF, high frequency; LF, low frequency.

\section{Serum Estradiol Vs HF}

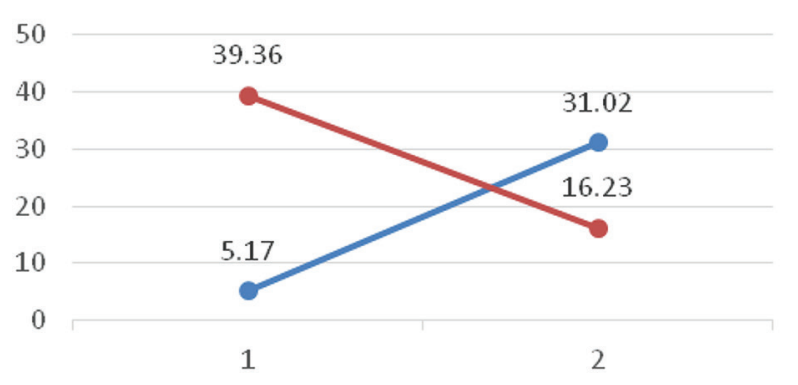

Fig. 1 Correlation of serum estradiol with the high-frequency (HF) variable of autonomic activity. reduced parasympathetic activation. ${ }^{23,24}$ Variations in heart rate and $\mathrm{BP}$ regarding various procedures performed are the hallmarks of AFT. Normally, even during rest, the duration of R-R intervals is not constant. HRV is generally assessed based on time- or frequency-domain analysis. Time-domain indices are used to evaluate the influence of various procedures on HRV over short periods too. ${ }^{25}$ Recent studies have shown that time- and frequency-domain indices can be reliably measured in a short-time series of 10 to 30 seconds. ${ }^{26}$ In our study, we have used short periods of measurement to see the influence of various stressful procedures on time- and frequency-domain indices such as rMSSD and the frequency-domain measure such as HF power. ${ }^{26} \mathrm{HF}$ is interpreted as a marker of parasympathetic activity and is influenced by 
the respiratory rate. LF is affected by both sympathetic and parasympathetic stimulations. In our study, we found high LF and low HF in obese individuals indicating high sympathetic activity and low parasympathetic activity that is detrimental to the health of the heart. The ratio of low to high frequency (LF:HF) shows the sympathovagal balance. Higher LF:HF values indicate increased sympathetic activity, and lower rates indicate increased vagal activity. In our study, we observed a high ratio in the nonobese and low ratio in obese postmenopausal women. ${ }^{27}$ However, a reanalysis by some researchers has shown that LF can be a marker of parasympathetic nervous system activity. ${ }^{27}$

Serum E2 levels are directly related to BMI in postmenopausal women. Fat tissue is the main source of circulating estrogens after menopause. High levels of estrogen in postmenopausal obese women are seen to reduce the HF variable of frequency-domain component indicating a low level of parasympathetic activity in these individuals making them prone to CVDs ( - Fig. 2). This study supports the fact that disturbances of autonomic activity with reductions in parasympathetic functions causes an inability to control the heart rate leading to high sympathetic activity associated with stress and CVDs.

Blood pressure changes were observed with sympathetic autonomic tests. In our study, we found that there was less increase in DBP in obese postmenopausal women with isometric handgrip activity indicating the deranged autonomic activity in this group, which is similar to previous studies. ${ }^{8}$ Also, a fall in DBP by $>10 \mathrm{~mm} \mathrm{Hg}$ with posture from supine to standing further confirmed the loss of autonomic adjustments in obese postmenopausal women.

This study suggests that postmenopausal women have weak autonomic activities and hence become prone to accelerated CVDs. Hence, it is necessary that these derangements be identified early during menopause and appropriate intervention be given to stop or delay any potential heart disease.

The main strength of this study is that it has shown the complete association of sympathetic and parasympathetic activity with the state of obesity and high extragonadal estrogen levels that are produced in obese postmenopausal women, which do not provide any cardioprotection.

FREQUENCY DOMAIN VARIABLES

IN OBESE AND NON-OBESE POST

MENOPAUSAL WOMEN

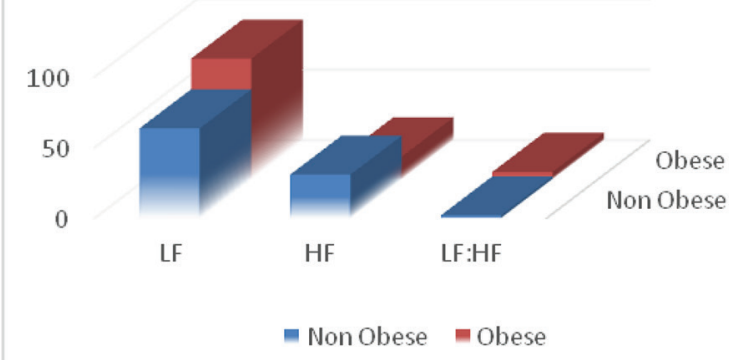

Fig. 2 Comparison of frequency-domain variable between nonobese and obese postmenopausal women.

\section{Conclusion}

Our study has broadly shown that postmenopausal women with obesity suffer from autonomic derangements and are possibly at an increased risk of developing heart disease. Screening women to detect any such derangement using reliable and precise tests of autonomic functions in an objective manner would help detect any abnormalities early. The information so obtained would translate into better clinical interpretation to detect any hidden or undiagnosed heart condition and improve the outcome through early and timely interventions. The tests can be performed with ease in a clinical setup, and we recommend utilizing the services of cardiophysiology laboratories for such purposes where the physiologist can measure and interpret the results with precision and accuracy and support the clinicians in their diagnosis. As the tests specifically determine changes pertaining to sympathetic and parasympathetic activities, it would also be possible to advise such women to take up lifestyle modifying interventions such as yoga or meditation as additional means of achieving a balance between the two components. However, this study still has some limitations that can be improved to make it more robust by increasing the sample size and taking into consideration several factors that can influence autonomic activity such as physical and mental activities and diet. Further studies on the subject can refine the findings to make it even more applicable in the clinical context.

\section{Funding}

Prajwalika Scholarship Programme (2018) of WINCARS association.

\section{Conflicts of Interests}

None.

\section{Acknowledgments}

We thank the study participants, Dr. Imran Ahmed Siddiqui, Specialist, Department of Biochemistry, ESIC Superspeciality Hospital, and his team for their support in the measurement of biochemical parameters.

\section{References}

1 Garcia M, Mulvagh SL, Merz CN, Buring JE, Manson JE. Cardiovascular disease in women clinical perspectives. Circ Res 2016;118(8):1273-1293

3 Mosca L, Barrett-Connor E, Wenger NK. Sex/gender differences in cardiovascular disease prevention: what a difference a decade makes. Circulation 2011;124(19):2145-2154

4 Mosca L, Linfante AH, Benjamin EJ, et al. National study of physician awareness and adherence to cardiovascular disease prevention guidelines. Circulation 2005;111(4):499-510

5 Bastien M, Poirier P, Lemieux I, Després JP. Overview of epidemiology and contribution of obesity to cardiovascular disease. Prog Cardiovasc Dis 2014;56(4):369-381

6 Kwaśniewska M, Pikala M, Kaczmarczyk-Chałas K, et al. Smoking status, the menopausal transition, and metabolic syndrome in women. Menopause 2012;19(2):194-201

7 Donato GB, Fuchs SC, Oppermann K, Bastos C, Spritzer PM. Association between menopause status and central adiposity measured at different cutoffs of waist circumference and waist-to-hip ratio. Menopause 2006;13(2):280-285 
8 van den Berg MP, Smit AJ. Bedside autonomic function testing in patients with vasovagal syncope. Pacing Clin Electrophysiol 1997;208 Pt $2: 2039-2042$

9 Hilz MJ, Dütsch M. Quantitative studies of autonomic function. Muscle Nerve 2006;33(1):6-20

10 Ewing DJ, Clarke BF. Diagnosis and management of diabetic autonomic neuropathy. Br Med J (Clin Res Ed) 1982;285(6346): 916-918

11 Torsvik M, Häggblom A, Eide GE, Schmutzhard E, Vetvik K, Winkler AS. Cardiovascular autonomic function tests in an African population. BMC Endocr Disord 2008;8:19-28

12 Kleiger RE, Miller JP, Bigger JT Jr, Moss AJ. Decreased heart rate variability and its association with increased mortality after acute myocardial infarction. Am J Cardiol 1987;59(4):256-262

13 Sayers BM. Analysis of heart rate variability. Ergonomics 1973;16(1):17-32

14 Pagani M, Lombardi F, Guzzetti S, et al. Power spectral analysis of heart rate and arterial pressure variabilities as a marker of sympathovagal interaction in man and conscious dog. Circ Res 1986;59(2):178-193

15 Malliani A, Pagani M, Lombardi F, Cerutti S. Cardiovascular neural regulation explored in the frequency domain. Circulation 1991;84(2):482-492

16 Carr MC. The emergence of the metabolic syndrome with menopause. J Clin Endocrinol Metab 2003;88(6):2404-2411

17 Ferrara CM, Lynch NA, Nicklas BJ, Ryan AS, Berman DM. Differences in adipose tissue metabolism between postmenopausal and perimenopausal women. J Clin Endocrinol Metab 2002;87(9):4166-4170

18 Rabbia F, Silke B, Conterno A, et al. Assessment of cardiac autonomic modulation during adolescent obesity. Obes Res 2003;11(4):541-548
19 Freitas IM, Miranda JA, Mira PA, Lanna CM, Lima JR, Laterza MC. Cardiac autonomic dysfunction in obese normotensive children and adolescents. Rev Paul Pediatr 2014;32(2):244-249

20 Nagai N, Matsumoto T, Kita H, Moritani T. Autonomic nervous system activity and the state and development of obesity in Japanese school children. Obes Res 2003;11(1):25-32

21 Kotchen TA. Obesity-related hypertension: epidemiology, pathophysiology, and clinical management. Am J Hypertens 2010;23(11):1170-1178

22 Huxley R, Mendis S, Zheleznyakov E, Reddy S, Chan J. Body mass index, waist circumference and waist:hip ratio as predictors of cardiovascular risk-a review of the literature. Eur J Clin Nutr 2010;64(1):16-22

23 Kimura T, Matsumoto T, Akiyoshi M, et al. Body fat and blood lipids in postmenopausal women are related to resting autonomic nervous system activity. Eur J Appl Physiol 2006;97(5):542-547

24 Maser RE, Lenhard MJ, Irgau I, Wynn GM. Impact of surgically induced weight loss on cardiovascular autonomic function: one-year follow-up. Obesity (Silver Spring) 2007;15(2):364-369

25 Pinna GD, Maestri R, Torunski A, et al. Heart rate variability measures: a fresh look at reliability. Clin Sci (Lond) 2007;113(3):131-140

26 Baek HJ, Cho CH, Cho J, Woo JM. Reliability of ultra-short-term analysis as a surrogate of standard 5-min analysis of heart rate variability. Telemed J E Health 2015;21(5):404-414

27 Reyes del Paso GA, Langewitz W, Mulder LJ, van Roon A, Duschek S. The utility of low frequency heart rate variability as an index of sympathetic cardiac tone: a review with emphasis on a reanalysis of previous studies. Psychophysiology 2013;50(5):477-487 